ten Sinn. Es handelt sich hier offenbar um einen Parallelfall zum "hypoxischen Paradoxon“ bei der Versorgung des Hirngewebes mit Sauerstoff ${ }^{9}$.

Es sei noch auf folgende Tatsache hingewiesen: bei der III. Gruppe, bei der der stärkste Aderlaß ausgeführt worden war, ist die Blutmenge des Aderlasses noch immer geringer als diejenige aller vorher verabreichten Blutinjektionen zusammen, selbst wenn man eine übernormale Abbauquote von $8 \%$ je Tag des injizierten Blutes mit berücksichtigt; trotzdem fallen die Erythrocytenwerte nach dem Aderlaß unter die Norm. Dies kann m. E. nur so gedeutet werden: bei der Injektion größerer Mengen von Erythrocyten kommt es, wie schon länger bekannt ist ${ }^{10}$, zu einer Eröffnung von zusätzlichen Kapillargebieten, die normalerweise nicht durchströmt werden. Offenbar bleibt nach einem Aderlaß, der aus dem Blut weniger Erythrocyten entfernt, als vorher injiziert worden war, ein Teil dieser Kapillaren offen, so daß durch die Blutverdünnung eine Unterschreitung der normalen 5-Mill.-Grenze bewirkt wird. Im Organismus solcher

9 E. Opitz u. N. S chneider, Ergebn. Physiol. 46, 126 [1950].

10 D. Grepp u. C. Wiggers, Amer. J. Physiol. 104, 423 [1933].
Tiere befindet sich demnach eine größere Gesamtzahl von Erythrocyten, als der Norm entspricht, bei einer verminderten Zellkonzentration. $\mathrm{Da}$ in diesen Fällen eine kxäftige Erythropoesesteigerung beobachtet wird, läßt sich folgern, daß es bei der Steuerung der Erythropoese im normalen Organismus und bei normaler Luftatmung nicht auf die Gesamtzahl der Sauerstoffträger, sondern auf ihre Konzentration in der Volumeneinheit ankommt.

Da ein kräftiger Aderlaß, der die Erythrocytenzahlen jedoch nicht unter die 5-Mill./cmm-Grenze fallen läßt (Gruppe I), keine Steigerung der Erythropoeseaktivität mit sich bringt, sondern erst ein solcher, bei dem die Erythrocyten diese Grenze unterschreiten, läßt sich folgern, daß eine Verdünnung der Erythrocytenkonzentration als solche noch keine Steigerung der Erythropoese bewirken kann. Erst ein zusätzlicher relativer Sauerstoffmangel setzt den Erythropoesemechanismus in Bewegung. Damit scheint bewiesen, daß es sich bei der Steuerung der 2 Mechanismen um 2 getrennte stoffliche Prinzipien handeln muß.

Frl. H. T e i pe l danke ich für ihre wertvolle Hilfe bei der Durchführung der Arbeit.

\title{
Zur Kinetik des Zerfalls \\ von Diazoessigester und Nitrosoalkyl-Harnstoffen an sauren bzw. basischen Wirkungsgruppen von ionenaustauschenden Permutoiden
}

\author{
Von W. Lautsch und W. Rothkegel \\ Aus dem Institut für organische Chemie der Freien Universität Berlin, Berlin-Dahlem \\ (Z. Naturforschg. 6 b, 365-369 [1951]; eingegangen am 9. Juli 1951)
}

\begin{abstract}
Kationenaustauscher und mit quartären Ammoniumgruppen ausgestattete Permutoide bewirken den Zerfall von Diazoessigester bzw. Nitrosomethylharnstoff. Eine Abhängigkeit der Zerfallsgeschwindigkeit konnte bei geringen Austauschermengen festgestellt werden, bei größeren Austauschermengen war jedoch eine Annäherung der Zerfallsgeschwindigkeitskonstanten an einen Grenzwert zu bemerken. Diese Beobachtungen können für Austauschersäulen von Interesse sein, da dort annähernd gleiche Versuchsbedingungen vorliegen.
\end{abstract}

1. Die katalytische Wirksamkeit von mit Sulfons äure-bzw. Carboxylgruppen ausgerüsteten Kationen-Austauschern auf den Zerfall von Diazoessigester

$\mathrm{V}$ or mehr als 40 Jahren zeigte B redig ${ }^{1}$, daß der Zerfall von Diazoessigester unter Bildung von Stickstoff und Glykolsäureester „zu den empfindlichsten Katalysen durch Wasserstoffionen gehört" und daß diese Reaktion nach einem Zeitgesetz 1. Ordnung verläuft.

Wir verwendeten diese Reaktion in der Absicht, eine Aussage machen zu können über die katalytische Wirksamkeit der Wasserstoffionen, die in den $\mathrm{SO}_{3} \mathrm{H}$ und $\mathrm{COOH}-G r u p p e n$ der Ionen-Austauscher enthalten sind.

1 G. B re dig u. W. Fränke l, Z. Elektrochem. angew. physik. Chem. 11, 525 [1905]. 
Als mit $\mathrm{SO}_{3} \mathrm{H}$-Gruppen ausgerüstete KationenAustauscher verwendeten wir die Amberlite IR 100 und IR 120 sowie den vorwiegend mit CarboxylGruppen ausgerüsteten Amberlite IR C 50, später auch Kationen-Austauscher auf Basis von Ligninsulfonsäure eigner Herstellung ${ }^{2}$.

Zur Messung des Diazoessigester-Zerfalles fanden die in bekannter Weise in die freie Säure durch Behandlung mit verdünnter Mineralsäure und anschlieBender sorgfältiger Neutralwaschung verwandelten Ionen-Austausch-Präparate in Warburg-Gefäßen Aufnahme; der seitliche Stutzen der Warburg-Gefäße nahm die wäßrige Diazoessigester-Lösung auf. Alle Versuche wurden bei konstanter Schüttelgeschwindigkeit und Temperatur unter Verwendung der manometrischen Methodik von $\mathrm{W}$ a r b u r g ${ }^{3}$ durchgeführt.

Die katalytische Wirksamkeit der so vorbehandelten Kationen-Austauscher wurde in Abhängigkeit von deren Einwaage, ausgedrückt in den Werten für die Grammäquivalente, wie man sie durch potentiometrische Titration mit Alkali ${ }^{4}$ erhält, untersucht. Das Verhältnis der jeweils verwendeten Mole Diazoessigester $\mathrm{zu}$ den Mengen an verwendeten Ionen-Austauschern in Grammäquivalenten betrug $1: 17,5-1: 440$. Nur innerhalb dieses Bereiches ließ sich die entwickelte Stickstoffmenge nach der Warburgschen Methodik messen. Versuche, die darauf abzielten, das Verhältnis zwischen Diazoessigester und wirksamen Gruppen im Hinblick auf die katalytische Wirksamkeit der letzteren zu verringern bzw. das Verhältnis umzukehren, scheiterten an der zu hohen Zersetzungsgeschwindigkeit, die sich meßtechnisch nicht mehr verfolgen ließ.

Wenn der Zerfall des Diazoessigesters an Permutoiden ebenfalls eine monomolekulare Reaktion darstellen sollte, so müßte für die zur Zeit $t$ jeweils gemessene Stickstoffmenge $N_{\mathrm{L}}$ und der Reaktionsgeschwindigkeitskonstanten $k$ folgende Beziehung gelten, wenn $N_{\mathrm{E}}$ die Stickstoffmenge am Ende der Reaktion ist:

$$
-\ln N_{\mathrm{E}}-N_{\mathrm{L}}=k t-\ln N_{\mathrm{E}} .
$$

Aus der Steigung der Geraden müßte sich die Reaktionsgeschwindigkeitskonstante

$$
k=\frac{2,3 \lg \left(N_{\mathrm{E}}-N_{\mathrm{L}}\right)_{2}-\lg \left(N_{\mathrm{E}}-N_{\mathrm{L}}\right)_{1}}{t_{2}-t_{1}}
$$

errechnen lassen.

2 W. L a u t s ch, Chemie 57, 149 [1944].

3 O. W a r b u r g, Biochem. Z. 152, 51 [1924] (vgl. auch H. A. K r e b s in "Die Methodik der Fermente" von C. O p p e $n$ h e i m e r, Leipzig 1929).

4 W. L a u t s c h, Cellulosechem. 22, 97 [1944].

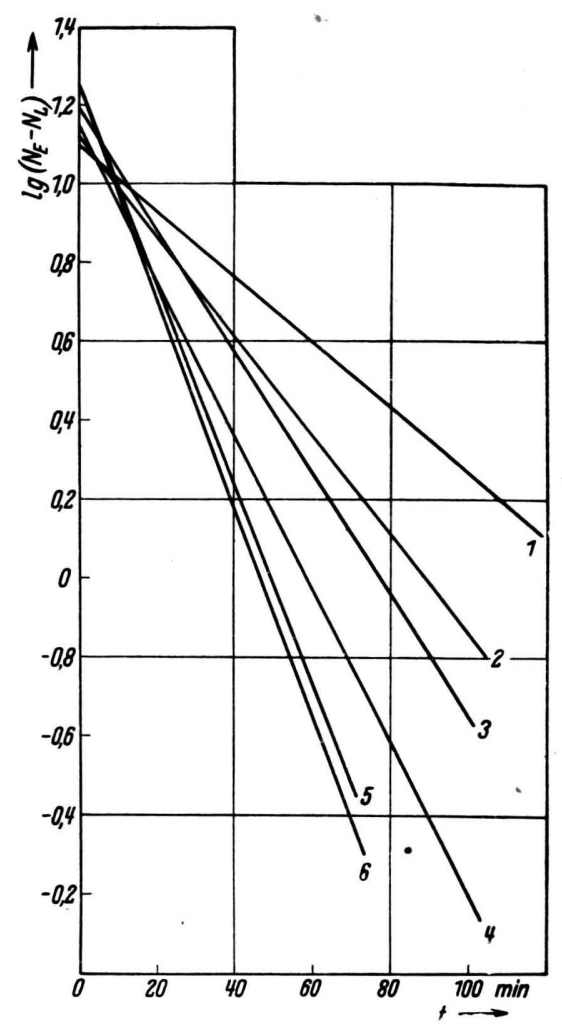

Abb. 1. Zerfall des Diazoessigesters an mit $\mathrm{SO}_{3} \mathrm{H}$ als Wirkungsgruppen ausgerüsteten Ionen-Austauschern (Amberlite IR 100 (Inhalt des Meßgefäßes etwa $18 \mathrm{~cm}^{3}$; je $1 \mathrm{~cm}^{3}$ einer wäßrigen Diazoessigester-Lösung, enthaltend $0,04 \cdot 10^{-4} \mathrm{~mol}$ Diazoessigester; verwendete Austauschermenge entspr. $0,74-9,6 \cdot 10^{-4}$ Val.; Temperatur $20^{\circ}$; Schüttelfrequenz $86 / \mathrm{min}$ ).

\begin{tabular}{|c|c|}
\hline $\begin{array}{c}\text { Kurve } \\
\text { Nr. }\end{array}$ & $\begin{array}{c}\text { Ionen-Austauschermenge in } \\
\text { Grammäquivalenten } \cdot 10^{-4}\end{array}$ \\
\hline 1 & 0,736 \\
2 & 1,348 \\
3 & 2,174 \\
4 & 3,712 \\
5 & 7,510 \\
6 & 9,614 \\
\hline
\end{tabular}

Ergebnisse: Trägt man als Abszisse die Zeit $t$ in Minuten, als Ordinate den Logarithmus der jeweils noch vorhandenen Menge an gebundenem Stickstoff $\left[\lg \left(N_{\mathrm{E}}-N_{\mathrm{L}}\right)\right]$ für den in der Abb. 1 wiedergegebenen Zerfall des Diazoessigesters an den mit $\mathrm{SO}_{3} \mathrm{H}$ Gruppen ausgerüsteten Amberlite IR 100 auf, wobei jede Kurve den Zerfall für die verschiedenen Mengen in Grammäquivalenten wiedergibt, so erhält man die in der Abb. 1 aufgezeigten Geraden, die eindeutig die Reaktion als eine solche 1. Ordnung kennzeichnen. 


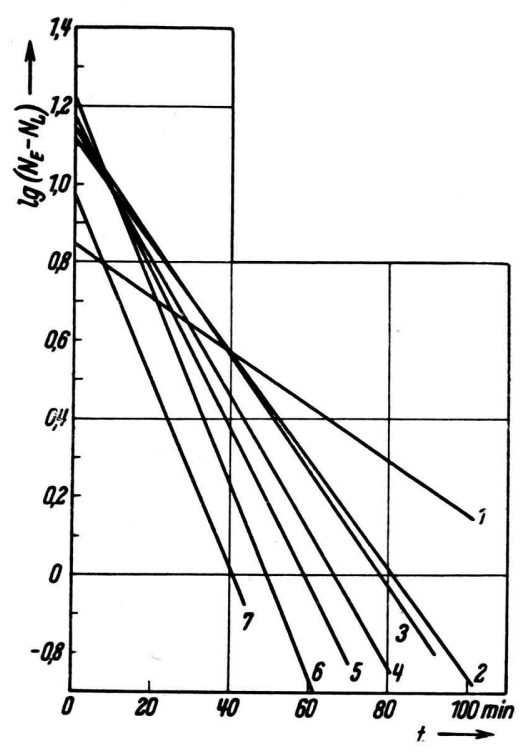

Abb. 2. Zerfall des Diazoessigesters an mit $\mathrm{SO}_{3} \mathrm{H}-\mathrm{Gruppen}$ als Wirkungsgruppen ausgerüsteten Ionen-Austauschern (Amberlite IR 120) (Versuchsbedingungen wie bei Abb. 1 angegeben).

\begin{tabular}{|c|c|}
\hline $\begin{array}{c}\text { Kurve } \\
\text { Nr. }\end{array}$ & $\begin{array}{c}\text { Ionen-Austauschermenge in } \\
\text { Grammäquivalenten } \cdot 10^{-4}\end{array}$ \\
\hline 1 & 1,39 \\
2 & 1,87 \\
3 & 2,05 \\
4 & 4,54 \\
5 & 6,25 \\
6 & 15,33 \\
7 & 14,31 \\
\hline
\end{tabular}

Analoge Ergebnisse wurden mit dem, durch einen höheren Gehalt an $\mathrm{SO}_{3} \mathrm{H}$-Gruppen als Wirkungsgruppen ausgezeichneten Amberlite IR 120 erhalten (Amberlite IR $100: 2,3 \mathrm{Val}$, Amberlite IR $120: 4,1 \mathrm{Val}$ pro $1000 \mathrm{~g}$ Austauscher), wie Abb. 2 zeigt.

Als Beispiel eines mit Carboxyl-Gruppen ausgerüsteten Ionenaustausch-Präparates verwendeten wir den Amberlite IR C 50. Die Ergebnisse sind in Abb. 3 eingetragen. Die geringere Neigung der Geraden läßt die sehr viel kleinere Reaktionsgeschwindigkeit an mit Carboxyl-Gruppen ausgerüsteten Permutoiden erkennen.

Ermittelt man aus der Steigung der in den Abb. 1 bis 3 wiedergegebenen Kurvenscharen die zugehörigen Reaktionsgeschwindigkeitskonstanten nach Gl. (2), so erhält man für die $k$-Werte der vermessenen Ionenaustausch-Präparate in allen 3 Fällen Kurven (vgl. Abb. 4), deren erster Abschnitt eine Linearität zwischen den verwendeten Ionen-Austauscher-Mengen, ausgedrückt in Grammäquivalenten, (Abszisse) und

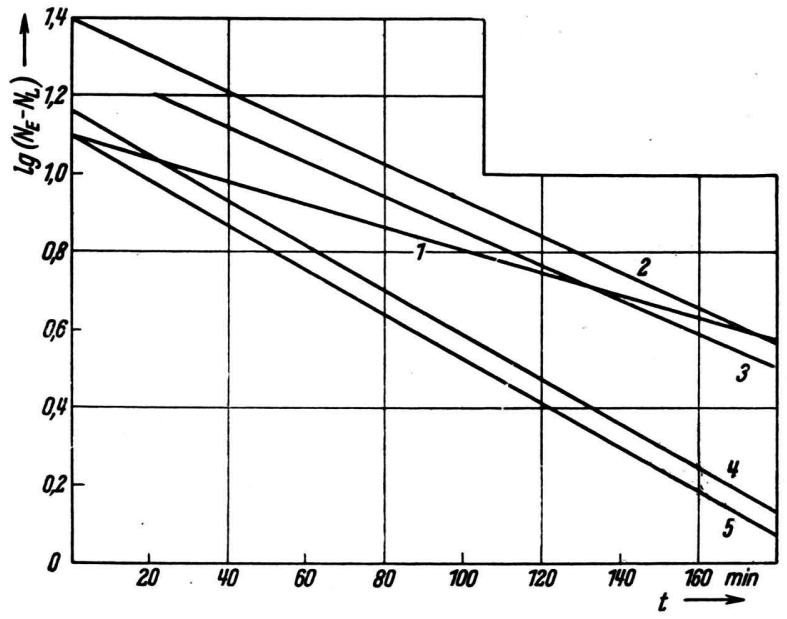

Abb. 3. Zerfall des Diazoessigesters an mit CarboxylGruppen ausgerüsteten Permutoiden (Amberlite IR C 50) (Versuchsbedingungen wie bei Abb. 1 angegeben).

\begin{tabular}{|c|c|}
\hline $\begin{array}{c}\text { Kurve } \\
\text { Nr. }\end{array}$ & $\begin{array}{c}\text { Ionen-Austauschermenge in } \\
\text { Grammäquivalenten } \cdot 10^{-4}\end{array}$ \\
\hline & \\
1 & 9,52 \\
2 & 22,20 \\
3 & 30,27 \\
4 & 41,21 \\
5 & 26,79 \\
\hline
\end{tabular}

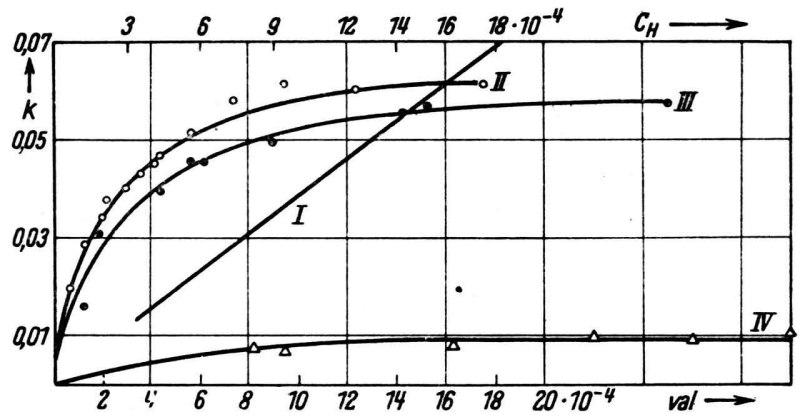

Abb. 4. $k$-Werte in Abhängigkeit von: $\mathrm{I}=$ der H-IonenKonzentration der zugegebenen Säure in Lösung nach B redig ${ }^{1}$; II = der zugesetzten Austauschermenge Amberlite IR $100\left(\mathrm{SO}_{3} \mathrm{H}\right)$; III $=$ der zugesetzten Austauschermenge Amberlite IR $120\left(\mathrm{SO}_{3} \mathrm{H}\right)$; IV $=$ der zugesetzten Austauschermenge Amberlite IR C $50(\mathrm{COOH})$.

der Reaktionsgeschwindigkeitskonstanten $k$ (Ordinate) erkennen läßt, welche sich anschließend in jedem Falle unabhängig von der Menge der verwendeten Ionenaustausch-Präparate einem Grenzwert nähern, während in Lösung nach $\mathrm{Bredig}$ in den $p_{\mathrm{H}^{-}}$ Bereichen, wo nachweislich keine Nebenreaktionen ${ }^{5}$

5 W. F r ä n k e l, Hoppe-Seyler's Z. physiol. Chem. 60, 202 [1907]. 
auftreten, Lineacität besteht (vgl. Kurve I; $C_{\mathrm{H}}=$ $\left.3-18 \cdot 10^{-4}\right)$.

Folgerungen und Deutung der Ergebn is s e: Der Zerfall des Diazoessigesters gehorcht an mit $\mathrm{SO}_{3} \mathrm{H}$ - und Carboxyl-Gruppen ausgerüsteten Permutoiden einem Zeitgesetz 1. Ordnung, wie es früher in Lösung von B redig gefunden wurde.

Die in den Abb. $1-3$ wiedergebenen Geraden lassen erkennen, daß Nebenreaktionen in nennenswertem Umfange nicht eintreten und daß der in Freiheit gesetzte Stickstoff in nachweisbarer Menge vom Austauscher nicht festgehalten wird. Man wird daraus schließen müssen, daß die katalytische Zersetzung sich im wesentlichen an der Oberfläche, weniger an den Wirkungsgruppen im Kapillarsystem des Austauschers vollzieht. Unter Berücksichtigung dieser Tatsache wird auch der asymptotische Verlauf der Kurven für die $\boldsymbol{k}$-Werte verständlich: Es kommt nach anfänglicher Linearität zwischen den $k$-Werten und der Zahl der Wirkungsgruppen bei weiterer Erhöhung der Austauscher-Menge zu einer Überschneidung der gegenseitigen Wirkungssphären der Austauscher-Partikelchen, so daß nach Überschreitung des Grenzwertes trotz Erhöhung der Austauschermenge eine Veränderung der Menge an wirksamen Gruppen offensichtlich nicht mehr eintritt. Dieser Befund ist für den Ablauf von Reaktionen an Austauscher-Säulen bemerkenswert, wo im allgemeinen die AustauscherPartikelchen keine Veränderung ihrer gegenseitigen Lage erfahren, Verhältnisse, wie sie bei der Auswertung der Zerfallsreaktionen in Warburg-Gefäßen unter den von uns gewählten Bedingungen bei größerer Austauschermenge ebenfalls annähernd gegeben sind. Durch den Schütteleffekt gerät im wesentlichen nur die die Austauscher-Partikelchen umgebende Lösung in Bewegung. In die Deutung fügt sich zwangsläufig die Beobachtung ein, daß eine stark beschleunigte Schüttelung der Meßgefäße, die die einzelnen Austauscher-Partikelchen voneinander unabhängig werden läßt, zu wesentlich höheren - allerdings nicht meßbaren - Zersetzungsgeschwindigkeiten führt.

An selbst hergestellten Kationen-Austauschern ${ }^{2}$, welche ohne Nachhärtung hochquellfähige Harzgele darstellen, ließ sich die oben wiedergegebene Gesetzmäßigkeit zwischen $k$-Werten und Grammäquivalenten infolge zu hoher Zersetzungsgeschwindigkeit des Diazoessigesters nicht beobachten. Offensichtlich findet die Zersetzung des Diazoessigesters hier auch an den Wirkungsgruppen im Innern der Harzgele statt. Dadurch treten Diffusionserscheinungen auf, welche die kinetische Deutung mit Hilfe der manometrischen Meßmethodik erschweren.

2. Der Zerfall von Nitrosomethylharnstoff an mit quartären AmmoniumGruppen ausgerüsteten Permutoiden

Dem katalytischen Zerfall des Diazoessigesters an den sauren Wirkungsgruppen von Ionen-Austauschern sollte z. B. der basische Zerfall von Nitrosotriacetonamin $^{6}$ entsprechen. Die Untersuchungen scheiterten an den hohen Zersetzungstemperaturen des Nitrosotriacetonamins, die eine saubere manometrische Messung in der Warburg-Apparatur verhindern.

Aus diesem Grunde untersuchten wir den Zerfall von Nitrosomethylharnstoff an den quartären Ammonium-Basen von Anionen-Austauschern, welcher in Lösung unter Bildung von Diazomethan und Alkalicyanat verläuft.

Es handelt sich hier um keine Katalyse, da die bei der Zersetzung entstehende Isocyansäure sich mit den quartären Wirkungsgruppen des Austauschers zu eirem unlöslichen Salz verbindet. Befinden sich jedoch die ionischen Wirkungsgruppen im großen Überschuß, so kann man auch hier ein Zeitgesetz 1. Ordnung erwarten.

Unsere Untersuchungen an mit quartären Ammoniumgruppen ausgerüsteten Permutoiden, welche in bekannter Weise durch Behandlung mit Alkali und nachfolgendem sorgfältigen Neutralwaschen in die festen quartären Ammoniumbasen verwandelt wurden, bestätigten diese Annahme. Das Verhältnis der verwendeten Menge an Nitrosomethylharnstoff zur Menge an Anionen-Austauschern, ausgedrückt in Grammäquivalenten, betrug 1:50-1:500.

Als Anionen-Austauscher, welche mit quartären Ammonium-Gruppen ausgerüstet sind, verwendeten wir den Amberlite IR A 400 sowie Präparate eigener Herstellung, wie sie durch Umsetzung von Polyäthylenimin mit Epichlorhydrin und tertiären Aminen (Pyridin, Nicotin u. a.) ${ }^{2}$ beschrieben wurde.

Die Zerfallsreaktion des Nitrosomethylharnstoffs wurde in Analogie zu der des Diazoessigesters nach der manometrischen Methode von Warburg untersucht. Da der Absorptionskoeffizient von Diazomethan in Wasser unseres Wissens nicht bekannt ist, ließ sich das gebildete Endvolumeñ Diazomethan nicht ermitteln. Wir verwendeten deshalb die in der

$6 \mathrm{Clibbens}$ u. Francis, J. chem. Soc. [London] 1912, 1358; F r a n c is u. G l a k e, J. chem. Soc. [London] 1913, 1722; Francis, Glake u. Roche, J. chem. Soc. [London] 1915, 1651. 


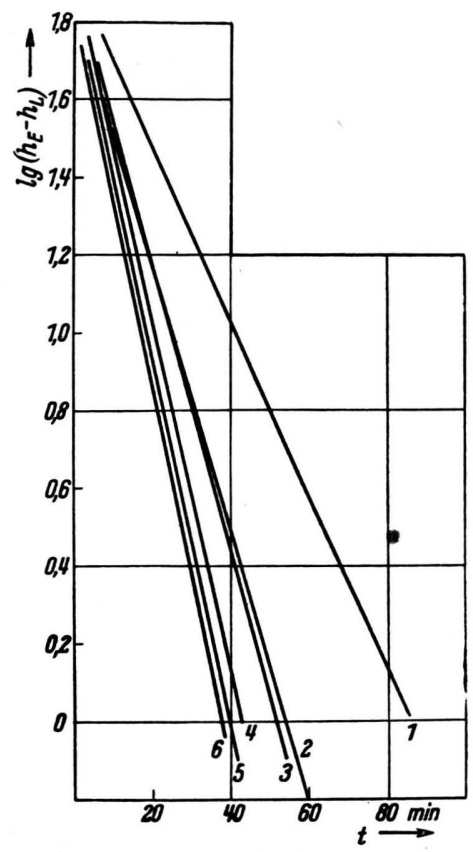

Abb. 5. Zerfall von Nitrosomethylharnstoff an mit quartären Ammoniumgruppen als Wirkungsgruppen ausgerüsteten Ionen-Austauschern (Amberlite IR A 400) (Inhalt des Meßgefäßes $18 \mathrm{~cm}^{3}$; Nitrosomethylharnstoff $0,0291 \cdot 10^{-4} \mathrm{~mol}$ je Versuch; verwendete Austauschermenge 1:6-13,2.10-4 Val; Temperatur $20^{\circ}$, Schüttelfrequenz $86 / \mathrm{min}$ ).

\begin{tabular}{|c|c|}
\hline $\begin{array}{c}\text { Kurve } \\
\text { Nr. }\end{array}$ & $\begin{array}{c}\text { Ionen-Austauschermenge in } \\
\text { Grammäquivalenten } \cdot 10^{-4}\end{array}$ \\
\hline 1 & 1,55 \\
2 & 9,50 \\
3 & 2,77 \\
4 & 15,41 \\
5 & 8,65 \\
6 & 13,15 \\
\hline
\end{tabular}

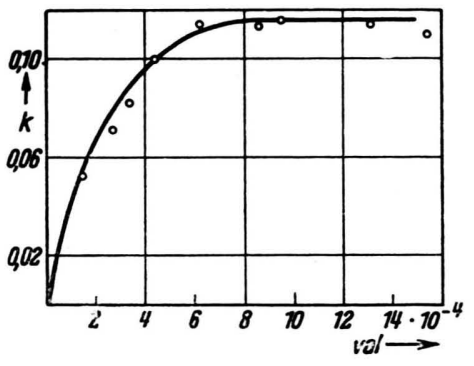

Abb. 6. $k$-Werte in Abhängigkeit von der angewendeten Austauschermenge Amberlite IR A $400\left(=\mathrm{N}^{(+)} \mathrm{OH}^{(-)}\right)$.

Zeiteinheit beobachtete Druckzunahme, d. h. den Anstieg der Flüssigkeitssäule, und trugen an Stelle des Ausdruckes $\lg \left(N_{\mathrm{E}}-N_{\mathrm{L}}\right)$ den Ausdruck $\lg \left(h_{\mathrm{E}}-h_{\mathrm{L}}\right)$ auf $\left(h_{\mathrm{E}}=\right.$ Manometerstand am Ende der Reaktion; $h_{\mathrm{L}}=$ Manometerstand zur Zeit $t$ ).

Abb. 5 zeigt, daß die Zerfallsreaktion des Nitrosomethylharnstoffs unter den von uns gewählten Bedingungen nach dem Zeitgesetz 1. Ordnung verläuft:

Aus der Steigung der Geraden ließen sich in bekannter Weise die Zerfallsgeschwindigkeitskonstanten $k$ in Abhängigkeit von der verwendeten Austauschermenge finden. Trägt man die so ermittelten $k$-Werte gegen die Grammäquivalente des Austauschers auf (Abb. 6), so erhält man einen analogen Kurvenverlauf, wie er beim Zerfall des Diazoessigsesters beobachtet wurde: nach anfänglicher Linearität nähern sich die $k$-Werte asymptotisch einem Grenzwert, der bei weiterer Erhöhung der Ionen-Austauschermenge keine Veränderung mehr erfährt.

Das Ergebnis ist analog zu deuten wie der Zerfall des Diazoessigesters an den sauren Wirkungsgruppen der Kationen-Austauscher.

\title{
Ein neues Verfahren zur Messung der optischen Aktivität
}

\author{
Von W. Broser und W. Lautsch \\ Aus dem Institut für organische Chemie der Freien Universität Berlin, Berlin-Dahlem \\ (Z. Naturforschg. 6 b, 369-372 [1951]; eingegangen am 21. Juni 1951)
}

Es wird ein Verfahren zur Bestimmung der optischen Drehung im Ultravioletten, Sichtbaren und nahen Ultraroten mittels Sekundärelektronenvervielfacher-Photozelle angegeben. Am Beispiel der Messung der Rotationsdispersion von Phäophorbid a und Methylphäophorbid a im Wellenlängenbereich von $740-1000 \mathrm{~nm}$ sowie der kinetischen Untersuchung der sehr rasch verlaufenden Mutarotation der Glucose in stark saurer Lösung werden die den bisherigen Methoden überlegenen Anwendungsmöglichkeiten des Verfahrens aufgezeigt.

$\mathrm{D}$ ie Messung der Drehung der Ebene des polarisierten Lichtes im Ultravioletten oder Infraroten erfolgt im allgemeinen auf photographischem ${ }^{1}$ Wege, während lichtelektrische Verfahren ${ }^{2}$ infolge der relativen Unempfindlichkeit der üblichen Photozellen bisher nur vereinzelt angewendet worden sind. Wegen 